\title{
Layer-wise control of selective laser melting by means of inline melt pool area measurements
}

Cite as: J. Laser Appl. 32, 022057 (2020); https://doi.org/10.2351/7.0000108

Submitted: 01 April 2020 . Accepted: 01 April 2020 . Published Online: 08 May 2020

Ema Vasileska, Ali Gökhan Demir (D), Bianca Maria Colosimo (D), and Barbara Previtali (D)

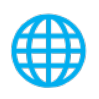

\section{Scilight Highlights of the best new research} in the physical sciences 


\title{
Layer-wise control of selective laser melting by means of inline melt pool area measurements
}

Cite as: J. Laser Appl. 32, 022057 (2020); doi: 10.2351/7.0000108

Submitted: 1 April 2020 . Accepted: 1 April 2020 .

Published Online: 8 May 2020

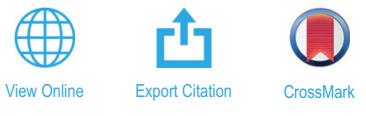

Ema Vasileska, Ali Gökhan Demir, (D) Bianca Maria Colosimo, (D) and Barbara Previtali (D)

AFFILIATIONS

Department of Mechanical Engineering, Politecnico di Milano, Via La Masa 1, 20156 Milan, Italy

Note: This paper is part of the Special Collection: Proceedings of the International Congress of Applications of Lasers \& Electro-Optics (ICALEO ${ }^{\circledR}$ 2019).

\begin{abstract}
Industrial selective laser melting (SLM) systems commonly employ a fixed set of process parameters throughout the build of the same component. The process parameters are generally found by experimental studies carried out on simple geometries which achieve high density. A common issue is related to the fact that the single set of parameters can be inadequate for small sections and overhang regions where thermal accumulation can occur. An online adaptation of process parameters is required for avoiding such issues and defects that commonly arise, such as the swelling phenomenon. A real-time control strategy would be desirable. However, the real-time control requires fast acquisition and reaction in the order of microseconds. Another approach is to provide corrective actions in a layer-wise fashion by elaborating the monitoring data collected during the previous layer. Therefore, this work proposes a layer-wise control strategy based on coaxial melt pool monitoring. For this purpose, an open SLM platform is employed, fitted with a complementary metal-oxide semiconductor camera, to view the process emission in the near infrared region. Initially, the nominal level of the melt pool area is defined on a simple geometry. Then, the melt pool area is monitored on more complex shapes. The melt pool area measured on each scan vector of a given layer is used to compensate the energy density of the same scan vector at the next layer. The results show an effective reduction of swelling defects on small geometries with fine details.
\end{abstract}

Key words: SLM, coaxial process monitoring, melt pool monitoring, pulse width modulation, process control

Published under license by Laser Institute of America. https://doi.org/10.2351/7.0000108

\section{INTRODUCTION}

Selective laser melting (SLM) is a metal additive manufacturing process that is achieving extensive industrial acceptance. Among others, benefits provided are the possibility for manufacturing complex geometries, lightweight structures, and internal channels to improve the product performance, which otherwise would be impossible with traditional manufacturing processes. SLM also provides means for producing high value products in small lots, which require defect-free manufacturing. Nevertheless, for a wider acceptance of SLM techniques in highly regulated industrial fields, such as aerospace or medical sectors, consistent quality and longterm performance of additively manufactured metallic parts are required to satisfy industrial standards and marketing needs. ${ }^{1-3}$

Most commonly, in both industrial and research environments, the same set of parameters is employed throughout the scanning of the whole workpiece. This set of scanning parameters is experimentally proven to result in a high-density fabrication of the part and is found by a number of trial-and-error testings that lead to a large consumption of raw materials, energy inputs, and time. However, this parameter set does not consider the part geometry or any potential in-process defects. The part geometry has a large impact on the melting conditions and the final quality of the workpiece. Examples for critical features in the geometry, which result in poor surface roughness, are overhangs and acute corners. ${ }^{4}$ In order to maintain the quality of the final workpiece, it is essential to implement process monitoring together with a feedback control of the scanning parameters that are regulated depending on the process output behavior.

Offline simulations of the melting process (FEM) combined with machine learning are giving the opportunity for open-loop optimization of process parameters, which theoretically achieve desired final properties. ${ }^{5,6}$ However, efficient simulation tools able to adapt process parameters depending on the part geometry are still to be developed. Implementing an effective closed-loop control solution with sensor feedback is still in the research phase for SLM 
technology. There are significant barriers for adoption of the realtime control in SLM, such as high sampling rates required to capture the fast solidification dynamics of the material, as well as lack of appropriate models for online estimation and control. ${ }^{7,8}$ According to Fox et al., ${ }^{9}$ it is sufficient to control melt pool dimensions throughout the entire process in order to ensure robust product qualities. Among the melt pool feedback controllers found in the literature, most of them target the melt pool area. A real-time feedback control has been employed by Kruth et al. ${ }^{10}$ and Craeghs et $a l .{ }^{11}$ on overhang structures and cubes using a photodiode and complementary metal-oxide semiconductor (CMOS) camera for measuring the melt pool indicators and varying the laser power based on the sensor signal. Yeung et al. ${ }^{12}$ implemented a highspeed coaxial camera on an SLM platform and presented a jerk-limited motion control for improving the position and velocity accuracy, as well as a thermal-adjusted strategy that locally varies laser power based on the adjacent solidified material. Renken et al. ${ }^{13}$ closed the loop in real time with a pyrometer implemented in a field programmable gate array environment and achieved reduction of the deviation of process temperature that led to more stable conditions in the melt pool. Throughout the research works, the potentiality of improving the quality and microstructure of the SLM fabricated part with adapting the process parameters by continuously observing the thermal behavior of the melting process is confirmed. ${ }^{14}$ Indeed, several machine manufacturers have started implementing a coaxial monitoring equipment in order to monitor the melt pool conditions. However, effective control strategies exploiting these sensors are yet to be developed. ${ }^{15-18}$

Therefore, this work proposes a novel control scheme based on coaxial monitoring and correction of energy density of each scanned vector of the next layer. In the initial part of the work, the layer-wise control concept is introduced along with candidate control strategies. Later, an experimental campaign is presented to build an empirical model linking the duty cycle and scan vector length to the melt pool area. Next, the use of the candidate control strategies is simulated on different geometries by estimating the melt pool area behavior with the empirical model. Finally, the most advantageous control method is validated by experimental runs.

\section{LAYER-WISE CONTROL CONCEPT}

In SLM, the implementation of a real-time control can be cumbersome due to the involvement of temporal and dimensional scale of the physical phenomenon. Accordingly, a layer-wise control strategy can be employed to correct or limit the propagation of the process defect among the successive layers. Swelling is one of the SLM defects, which is defined as deposited material extruding from the powder bed due to excessive thermal accumulation. Resultantly, the geometrical accuracy is compromised. These regions can wear the powder recoater or eventually be stripped off by the recoater. Pointy edges, thin sections, overhang regions, or poorly supported sections are more prone to heat accumulation and hot spot formation, where swelling phenomenon can be more critical. ${ }^{19}$

The energy input can be managed in order to avoid the swelling phenomenon. The laser energy density $E$ is a common parameter used to define the energy delivered at unit volume material and is defined as

$$
E=\frac{P_{p e a k} \cdot \delta}{h \cdot z \cdot v}
$$

where $h$ is the hatch distance, $z$ is the layer thickness, $v$ is the scanning speed, $P_{\text {peak }}$ is the peak laser power, and $\delta$ is the duty cycle. For systems operating at continuous wave (CW) emission, duty cycle $\delta$ equals to 1 , while in the systems operating with pulsed wave (PW) emission by power modulation, the duty cycle varies. ${ }^{20}$ For a given material, these process parameters are commonly set to achieve high density (i.e., >99\%) on simple geometries such as cubical samples, and variations according to the part geometry is usually not considered. Therefore, the adaptability of the same process parameters to both bulky parts and fine structures is limited. Assigning different process parameters to different parts, as well as different sections of the same part, is possible to most of the industrial SLM systems (see Fig. 1). Varying process parameters within the layer in order to avoid heat accumulation is a promising concept that requires further studies.

From this point of view, controlling the energy density by modulating the laser power at the heat accumulation zones, and hence the swelling regions, is the core of the layer-wise control strategies. Figure 2 shows a schematic representation of the proposed control strategy. In order to reveal the heat accumulation zones, coaxial monitoring of the melt pool via a CMOS camera is the chosen method. The reference melt pool size is determined through the common practice of producing samples with simple shapes such as cubes with process parameters determining the desired density. The control method aims to adjust the duty cycle in order to maintain the melt pool size at the correct value.

In the layer-wise control concept, the scanning process of the preceding layer is evaluated in order to correct the melting condition in the following layer at the points where it deviates from the desired behavior. For analyzing the correctness of the scanning process, the melt pool area (MPA) is considered as a process signal, which indicates the thermal behavior during the process. By monitoring the melt pool, the amount of laser energy delivered to the part can be indirectly observed. If an excess or a lack of energy input is observed, the scanning parameters need to be modified in order to stabilize the process.

The layer-wise control strategy works such that it calculates and evaluates the average melt pool area of the scan vector $k$ in layer $i$ and corrects the delivered energy density in the same scan vector $k$ in the subsequent layer $i+1$, by adjusting the duty cycle of the laser power. In the case when the melting process of a scan vector in the previous layer is optimal, then the equivalent duty cycle will be employed likewise in the following layer. In this way, the faulty melting conditions are instantaneously corrected and the defect propagation in the height of the build is avoided.

\section{A. Candidate strategies for the layer-wise control}

Among several possibilities, two different approaches were examined for the layer-wise control, with a fixed and with an adaptive reference point. In both methods, the melt pool area was employed as a process variable, and the duty cycle of the laser power as a 


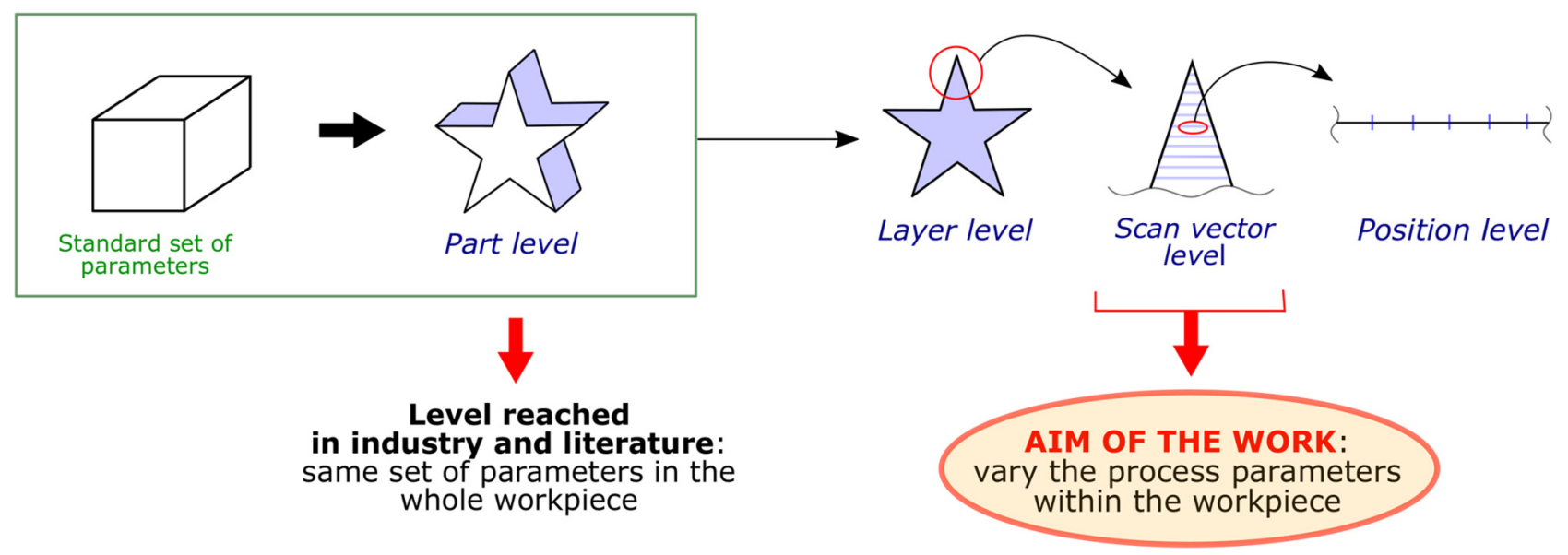

FIG. 1. Variation of process parameters at different levels.

process parameter. The error of the melt pool area of the scan vector $k$ in layer $i\left(e_{k, i}\right)$ is computed as the difference between the nominal melt pool area $\left(M P A_{n o m}\right)$ and the melt pool area $\left(M P A_{k, i}\right)$ acquired when scanning the same scan vector $k$ and layer $i$. Depending on the value of the error, a negative sign indicates underheated melting process, while a positive error indicates an overmelting phenomenon,

$$
e_{k, i}=M P A_{n o m}-M P A_{k, i}
$$

The difference between the two approaches for the layer-wise control is the method for calculating the corrected duty cycle $\delta_{k, i+1}$ as a function of the error of the melt pool area $e_{k, i}$ and the duty cycle employed in the previous layer $\delta_{k, i}$ for the corresponding scan vector $k$. The control schemes of the layer-wise control strategies are shown in Figs. 3 and 4 respectively, where $M P A$ is the melt pool area of the melt pool $M P$ acquired with the camera and obtained from the image analyses algorithm, and $d$ are the disturbances in the process that can be a result of the process itself or of the powder material, gas flow, or the recoating system.

When the layer-wise control with fixed reference point is considered, the adjusted duty cycle $\delta_{k, i+1}$ is found as a sum of the previous duty cycle $\delta_{k, i}$ and a correction factor $\Delta \delta_{k, i+1}$. The correction factor $\Delta \delta_{k, i+1}$ is computed as a function of the error of the melt pool area $e_{k, i}$,

$$
\delta_{k, i+1}=\delta_{k, i}+\Delta \delta_{k, i+1}\left(e_{k, i}\right)
$$

In the control strategy with adaptive reference point, the reference point of the duty cycle is modified between $\delta_{\text {ref,max }}$ and $\delta_{\text {ref,min }}$

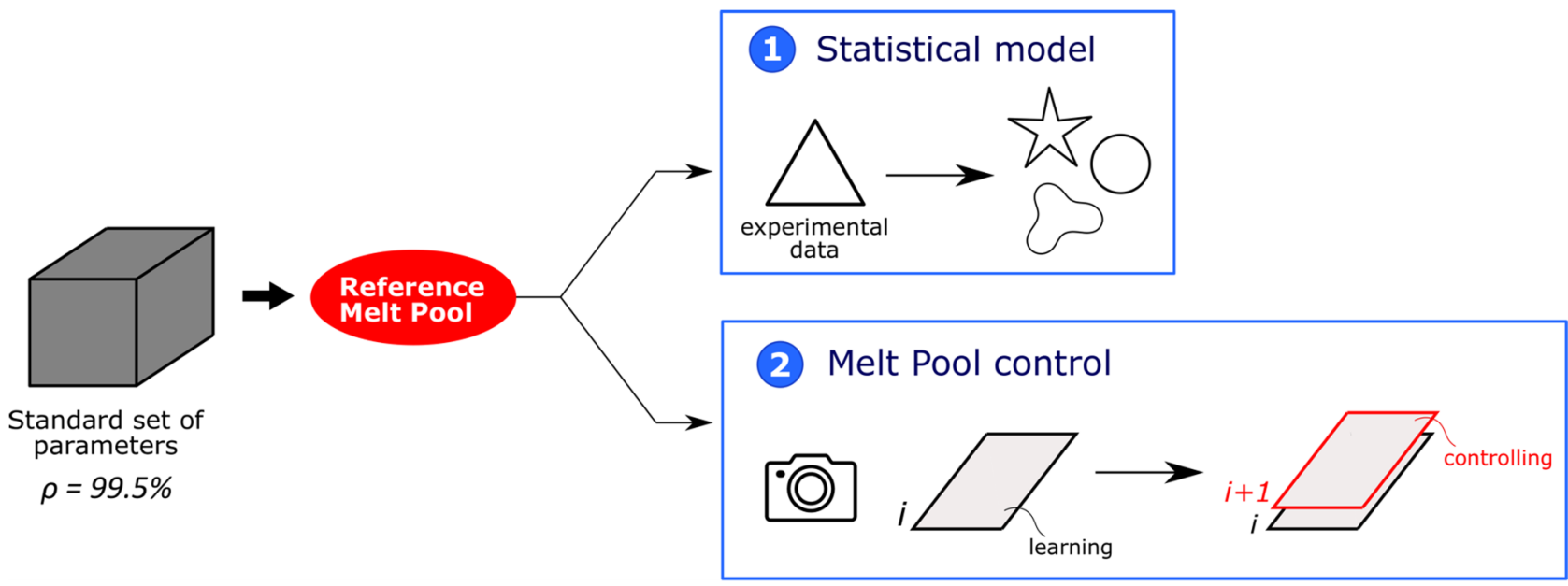

FIG. 2. Schematic representation of the layer-wise control strategy. 
a)

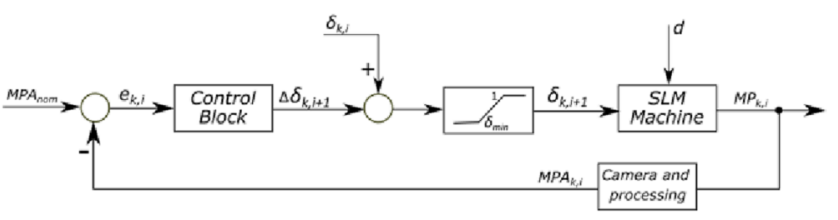

b)

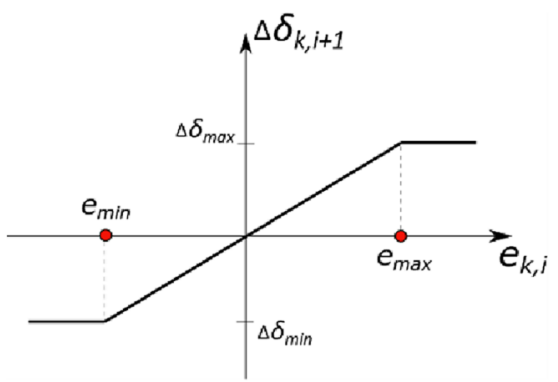

FIG. 3. Layer-wise control with fixed reference point: (a) control scheme and (b) control graph.

a)

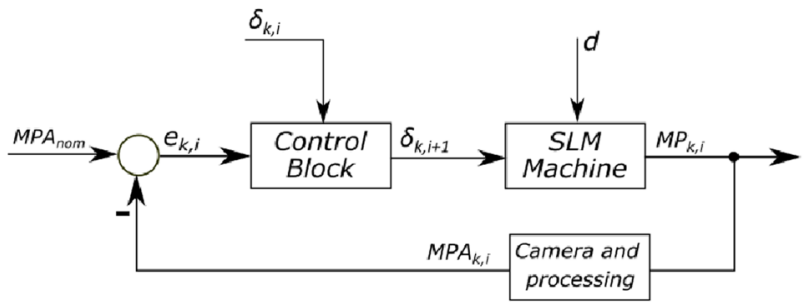

b)

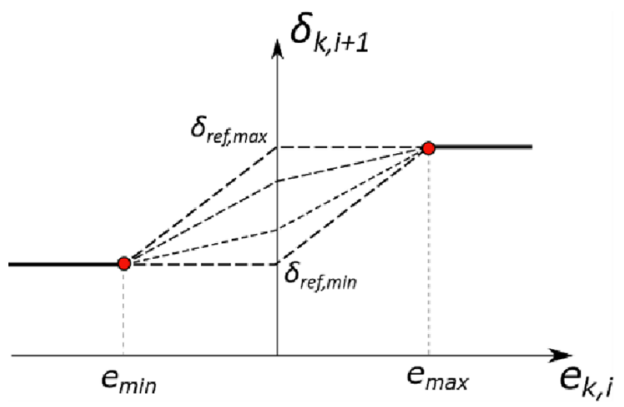

FIG. 4. Layer-wise control with adaptive reference point: (a) control scheme and (b) control graph with variable reference point. in the control graph [Fig. 4(b)] at each scan vector of every layer. For a given layer and scan vector, the duty cycle of the previous layer is taken as the reference point $\left(\delta_{k, i}=\delta_{r e f, k, i+1}\right)$. Then, the control graph is adjusted to constitute two linear relationships in the negative and positive MPA error regions. The duty cycle is calculated using these adapted relationships for $e_{k, i}$. The control graph is, therefore, adapted for each vector and layer.

\section{B. Experimental inputs and strategy choice}

Both the control strategies require experimental inputs. The reference melt pool area is determined by printing a simple bulk geometry scanned with nominal process parameters. The experimental model is derived to define the maximum $\left(e_{\max }\right)$ and minimum $\left(e_{\text {min }}\right)$ error levels as well as the duty cycle levels. A straightforward approach to investigate the thermal accumulation behavior is to vary the scan length $\left(l_{s v}\right)$ at each scanned layer with the use of triangle geometry. Shorter vectors are more prone to thermal accumulation due to the reduced duration between consecutive vectors. Hence, the experimental campaign was designed to evaluate the influence of the duty cycle and the scan vector length on the melt pool area. The measured data were used to fit the empirical model linking the melt pool area (response) as a function of the duty cycle and the scan vector length. This transfer function was then used for experimental validation.

\section{EXPERIMENTAL SETUP}

\section{A. Open selective laser melting platform}

An open and custom-made SLM platform, namely, Penelope was used to conduct the experimental work. The system is characterized by a working volume of $60 \times 60 \times 20 \mathrm{~mm}^{3}$ and the ability to process small amounts of the powder. The powder bed is placed in a closed chamber where an inert atmosphere is created. The laser source employed in the experiment is a single mode fiber laser (IPG Photonics YLR-150/750-QCW-AC, Cambridge, MA, USA) with a maximum power of $250 \mathrm{~W}$, and it can emit in CW and can be modulated to emit at the PW regime with a maximum modulation frequency of $10 \mathrm{kHz}$. The laser optical chain consists of a collimating unit with a focal length of $50 \mathrm{~mm}$, a focus shifting two-lens system (VarioScan 20, Scanlab, Puchheim, Germany), and a $420 \mathrm{~mm} \mathrm{f}$-theta lens, while the deflection of the laser beam toward the building platform is achieved using two galvanometric mirrors. The scanning parameters and trajectory are set using SCANMASTER software (Cambridge Technologies, Bedford, MA), whereas the control of the movement of the mechanical system and the chamber pressure are employed in the LABviEW environment

TABLE I. Main characteristics of the open SLM platform.

\begin{tabular}{lc}
\hline \hline Parameter & Value \\
\hline Laser emission wavelength, $\lambda$ & $1070 \mathrm{~nm}$ \\
Max. laser power, $\mathrm{P}_{\max }$ & $250 \mathrm{~W}$ \\
Beam quality factor, $\mathrm{M}^{2}$ & 1.1 \\
Nominal beam diameter on focal plane, $\mathrm{d}_{0}$ & $70 \mu \mathrm{m}$ \\
\hline \hline
\end{tabular}


(National Instruments, Austin, TX). The main specifications of the open SLM platform and its optical chain can be found in Table I.

\section{B. Material}

Gas-atomized stainless steel AISI 316L (Cogne Acciai, Brescia, Italy) powder was used throughout the work. The powder packing density was $4.07 \mathrm{~g} / \mathrm{cm}^{3}$, while the powder size distribution was measured as $\mathrm{D}_{10}: 22.9 \mu \mathrm{m}, \mathrm{D}_{50}: 31.9 \mu \mathrm{m}$; and $\mathrm{D}_{90}: 44.3 \mu \mathrm{m}$.

\section{Monitoring module}

The monitoring module was mounted between the scanner head and the focus variation optics (see Fig. 5). The melting zone was coaxially viewed through a dichroic mirror reflective between 400 and $1000 \mathrm{~nm}$. A $120 \mathrm{~mm}$ focusing lens (Camera Adapter, Scanlab GmbH, Puchheim, Germany) was used to fix the image plane with a focal length of $120 \mathrm{~mm}$. As the laser beam scans the powder, the process emission is reflected back through the f-theta lens, the galvanometric mirrors and the dichroic mirror toward the camera sensor. Furthermore, implementing different optical filters along the path of the reflected process light can capture various desired emission bands. Thermal emission images between the range of 850 and $1000 \mathrm{~nm}$ were acquired by employing two optical filters in order to view only the near infrared (NIR) region (FELO850 and FSH1000, Thorlabs, Newton, NJ, USA). An industrial CMOS camera (Ximea xiQ USB Vision, Münster, Germany) was installed, with a sensor size of $1280 \times 1204$ pixel $^{2}$ and a pixel size of $4.8 \times 4.8 \mu \mathrm{m}^{2}$. By adapting the region of interest, the field of view was adjusted to $4.3 \times 4.3 \mathrm{~mm}^{2}$ with a spatial resolution of $14 \mu \mathrm{m} /$ pixel, which permits an acquisition rate of $1200 \mathrm{fps}$.

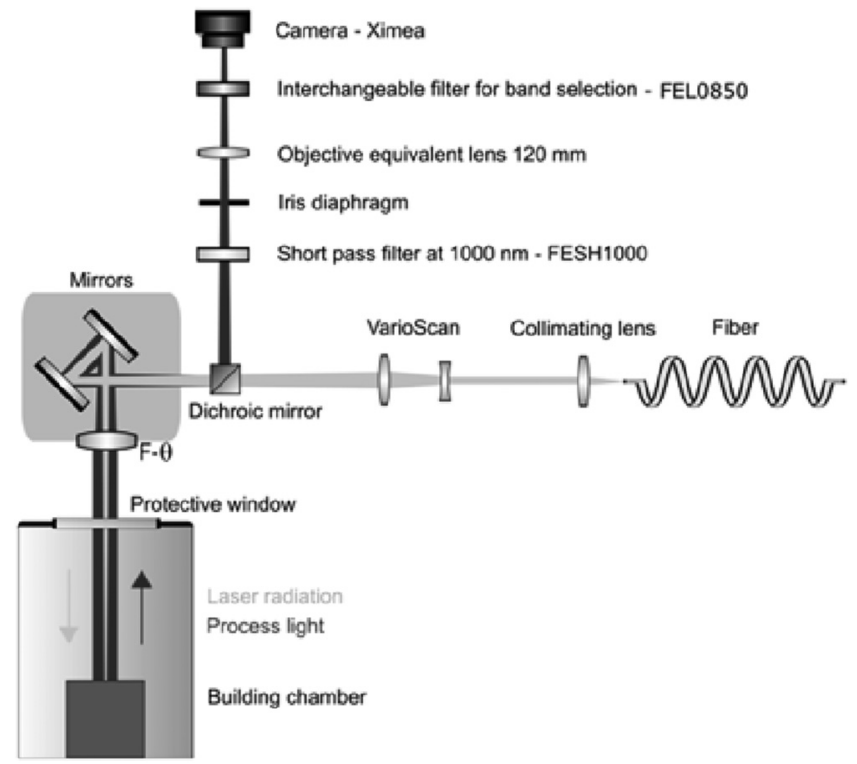

FIG. 5. Optical chain of the coaxial monitoring setup employed in the experiments.
The monitoring module provides a compromise between spatial and temporal resolution. With the given frame rate and a scan speed of $400 \mathrm{~mm} / \mathrm{s}$ used in the experimental part of this work, the melt pool is observed at a distance of $332 \mu \mathrm{m}$ between each frame. The total duration of capture, data transfer, data processing, and the reaction phases was calculated as approximately $1.4 \mathrm{~ms}$. This duration exceeds the period between each frame $(0.83 \mathrm{~ms})$ and corresponds to a spatial displacement of $560 \mu \mathrm{m}$. Within the given conditions, the use of a real-time control system is not feasible. Hence, the use of layer-wise control strategies becomes more advantageous.

\section{Measurement of the melt pool area}

After obtaining the NIR images, an image processing algorithm was used to measure the melt pool area. In the present work, the focus was put on the melt pool area as a key indicator for process stability.

The molten pool size is estimated performing static thresholding on thermal emission images of the molten pool, using an in-house developed MATLAB routine. ${ }^{21}$ Thresholded images are binary matrices whose elements are 1 or 0 based on whether their original values were higher (or equal) or smaller than the threshold value. The threshold constant $(C)$ was set matching the area measurements with an external illuminator and the area computed from the NIR images. The ejected particles and spatters were isolated from the main melt pool.

In the abovementioned image processing procedure, the melt pool area is defined as the sum of the pixels above a certain brightness level, without considering spatter ejection blobs, multiplied by the square of the spatial resolution. The melt pool area of frame number $k$ can be calculated using the following equation:

$$
M P A_{k}\left(\mathrm{~mm}^{2}\right)=r^{2} \cdot \sum_{i=1}^{m} \sum_{j=1}^{n} p_{k_{i, j}}^{T},
$$

where $r$ is the spatial resolution, $p_{k_{i j}}$ is the gray level of the pixel in row $i$ and column $j$ of the frame $k$, and $p_{k_{i, j}}^{T}$ is the binary value of $p_{k_{i, j}}$ according to the threshold constant $C$, calculated as follows:

$$
p_{k_{i, j}}^{T}= \begin{cases}1, & p_{k_{i, j}} \geq C, \\ 0, & p_{k_{i, j}}<C .\end{cases}
$$

The measured MPA at each frame was assigned to the spatial position on the scan plane by means of a normalized crosscorrelation algorithm. In the absence of the processing laser, the scan pattern was executed with the use of an external illuminator, and the powder bed was observed with the coaxial monitoring module. The normalized cross-correlation function was used on these images to calculate the relative displacement between frames. By iteratively employing this procedure on consecutive frames, a position map for each layer can be reconstructed. ${ }^{22}$ 
TABLE II. Fixed and varied scanning parameters employed in the experiment.

\begin{tabular}{lc}
\hline \hline Fixed parameters & \\
\hline Layer thickness $(\mu \mathrm{m})$ & 50 \\
Hatch distance $(\mu \mathrm{m})$ & 70 \\
Scan speed $(\mathrm{mm} / \mathrm{s})$ & 400 \\
Peak power $(\mathrm{W})$ & 200 \\
Modulation frequency $(\mathrm{kHz})$ & 3 \\
Scan direction $\left(^{\circ}\right)$ & 0 \\
Scan strategy & Serpentine \\
Varied parameters & \\
\hline Duty cycle & $0.3-1.0$ \\
Scan vector length $(\mathrm{mm})$ & $0.05-5.0$ \\
\hline \hline
\end{tabular}

\section{EMPIRICAL MODEL OF THE MELT POOL AREA AS A FUNCTION OF SCAN LENGTH AND DUTY CYCLE}

\section{A. Experimental design}

A square geometry with $5 \mathrm{~mm}$ side was scanned in the CW regime for the purpose of finding the nominal melt pool area that is signifying a stable melting process, as it was experimentally proven to result in a high-density solid object with $\rho>99.5 \%$. Melt pool videos were acquired throughout the build.

An equilateral triangle with $5 \mathrm{~mm}$ side was chosen as the test geometry in order to investigate how the scan vector length affects the melt pool area, since it is a simple shape with a continuously changing scan vector length. The effect of the amount of energy input on the melt pool is investigated by varying the duty cycles from 0.3 to 1 . Each triangle was scanned starting from the longest scan vector and ending with the shortest scan vector at the edge of the triangle. The peak laser power was fixed to $200 \mathrm{~W}$ and the scanning velocity to $400 \mathrm{~mm} / \mathrm{s}$, while the hatch distance and layer thickness were $70 \mu \mathrm{m}$ and $50 \mu \mathrm{m}$, respectively. These process parameters are confirmed through preliminary experiments to successfully fabricate a part obtaining high density when the laser is scanning in the CW regime. Thirty layers were executed without changing the scan direction between layers. Two replicates were produced for all conditions. Table II summarizes the fixed and varied set of parameters used in the experiment.

\section{B. Melt pool analysis}

The nominal melt pool area was determined at $0.46 \mathrm{~mm}^{2}$ observing the monitoring images of the square shaped sample. Figure 6 shows the melt pool area maps on the triangle specimens as a function of the duty cycle. It can be concluded that the melt pool area is highly affected by the duty cycle, which, combined with the scan vector length, is basically determining the average laser power that is locally used. The duty cycles of 0.4 and 0.3 resulted in undermelting of the part throughout the whole scan, and the scan vector length had no influence on the melt pool area. When scanning with duty cycles $>0.4$, there was an increasing trend of the melt pool area as the scan vector length decreased. Toward the end of each triangle, an enlargement of the melt pool area was observed,
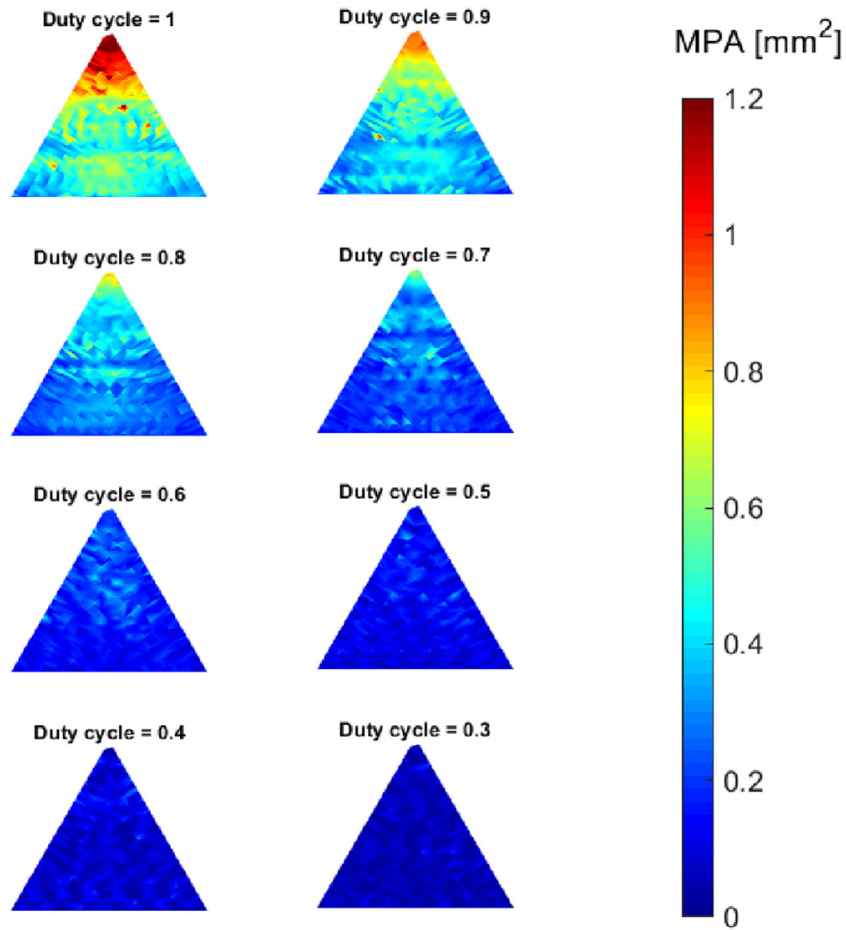

FIG. 6. Position-domain color maps of the melt pool area per duty cycle in triangular shaped workpiece, layer no 20.

as the scan vector was getting shorter and there was less time for heat dissipation, which resulted in larger overheating.

The high values of the melt pool area in the larger duty cycles occurred on the same position throughout the layers, thus creating a whole volume area where anomalous melt pools were detected. This indicated the occurrence of unwanted process phenomena during the building process, which influenced the building also in the following layers. In the areas where the overmelting phenomenon was present, swelling was eventually observed on the built specimens.

\section{Developed empirical model}

The monitoring images were analyzed to calculate the average MPA for each scan vector length and duty cycle combination. Conditions corresponding to duty cycles lower than 0.5 were excluded from the analysis, since these conditions corresponded to undermelting without a significant change on the measured melt pool area. A regression model was fitted to the experimental data with the following mathematical expression:

$$
\begin{aligned}
\ln (M P A)= & -3.0153+3.843 \cdot \delta-0.2241 \cdot l_{s v}-0.860 \cdot \delta^{2} \\
& +0.00912 \cdot l_{s v}^{2} .
\end{aligned}
$$

The model was found to fit the experimental data well with $R_{a d j}{ }^{2}=98.61 \%$, while normality and homogeneity of the residuals 


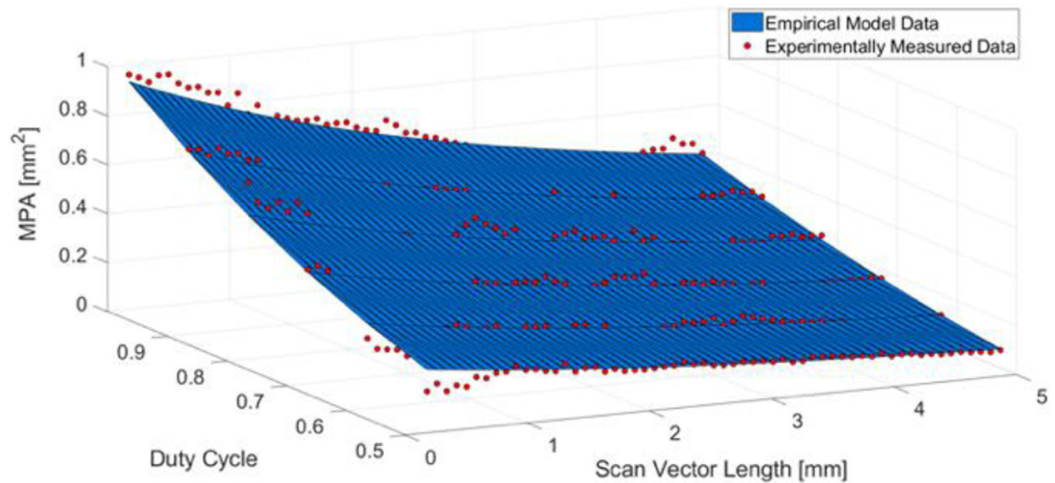

FIG. 7. Melt pool area as a function of scan vector length and duty cycle with a comparison between experimental data and the fitted model. were verified. A comparison between the experimental data and the data fitted with the model is presented in Fig. 7.

\section{SELECTION OF THE CONTROL STRATEGY}

\section{A. Experimental constants}

The data coming from the experimental runs were used to determine the experimental constants. It was observed that the duty cycles $<0.5$ generated undermelting conditions, where the limit condition for $e_{\max }=0.30 \mathrm{~mm}^{2}$. Overmelting conditions were defined for $e_{\min }=-0.48 \mathrm{~mm}^{2}$. The duty cycle was varied between $\delta_{\min }=0.6$ and $\delta_{\max }=1.0$ for the adaptive reference point. Analogously, the duty cycle was compensated in the fixed reference point with $\Delta \delta_{\text {min }}=-0.4$ and $\Delta \delta_{\max }=0.4$.

\section{B. Simulation of the control strategy responses}

The empirical model was used to simulate the response of the proposed control strategies. The average melt pool area was calculated for two different geometries. Tests were done on two simple geometries with pointy edges prone to thermal accumulation. Figure 8 shows the triangle and 4-point star chosen for the simulations. In the simulations, the first layer was scanned with two extreme duty cycles (1 and 0.6$)$. The data of the melting process of the first layer, which is used by the control strategy to learn how to modify the laser power in the next layer, is real data acquired when the exact geometry with the specified duty cycle is scanned. Starting from the second layer, the scanning of the following layers was simulated with controlled parameters. Here, the laser power of each scan vector of the subsequent layer was adapted depending on the melt pool area
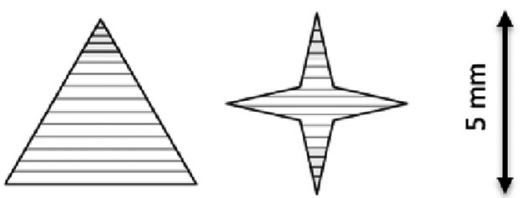

FIG. 8. Simulation geometries. generated by the empirical model at the same scan vector in the previous layer.

Figure 9 compares the average melt pool area achieved by the two different control schemes as a function of layer number, starting duty cycle, and scanned geometry.
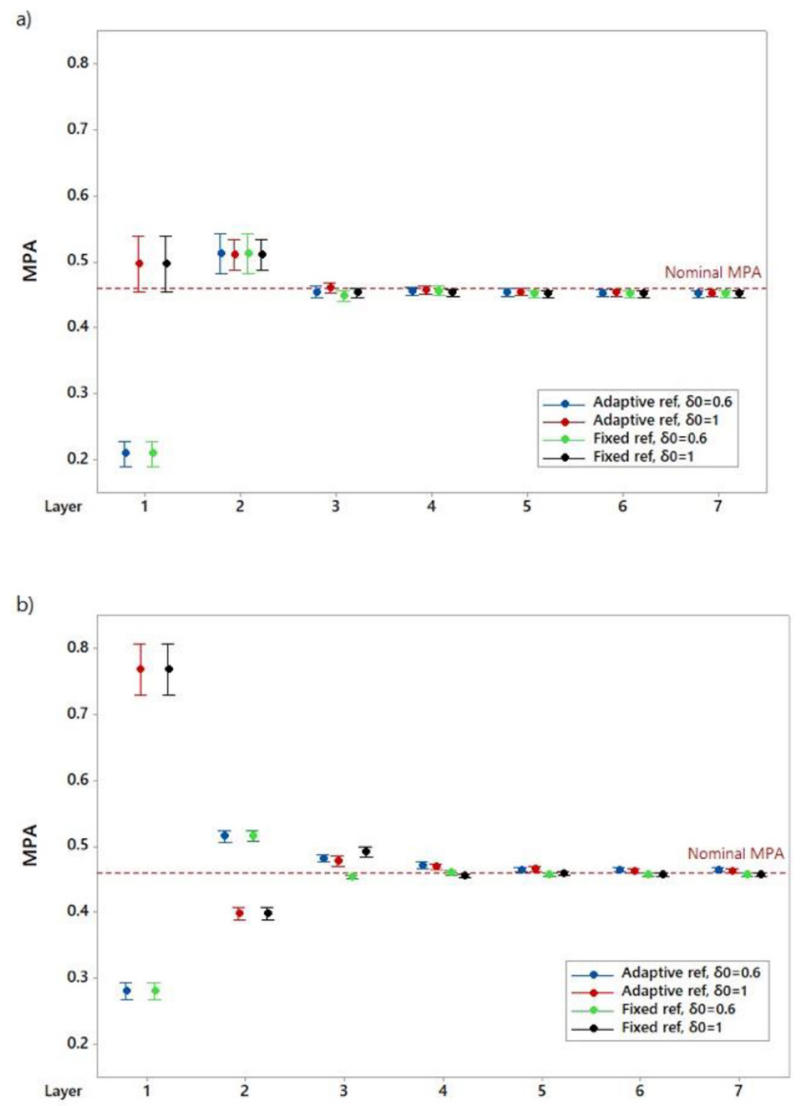

FIG. 9. Simulation and comparison of the time response of the two layer-wise correction strategies: (a) triangle-shaped workpiece and (b) star-shaped workpiece. 
TABLE III. Fixed and varied parameters employed in the validation experiments.

\begin{tabular}{lc}
\hline \hline Fixed parameters & \\
\hline Layer thickness $(\mu \mathrm{m})$ & 50 \\
Hatch distance $(\mu \mathrm{m})$ & 70 \\
Scan speed $(\mathrm{mm} / \mathrm{s})$ & 400 \\
Power $(\mathrm{W})$ & 200 \\
Modulation frequency $(\mathrm{kHz})$ & 3 \\
Scan direction $\left(^{\circ}\right)$ & 0 \\
Scan strategy & Serpentine \\
Initial duty cycle & 1 \\
Varied parameters & \\
\hline Control strategy & None; layer-wise \\
\hline \hline
\end{tabular}

When the initial layer is scanned in the CW mode $(\delta=1)$, the peaks of the workpieces start to overheat. After the control was employed, a small overshoot around the first layers was noticed in both control approaches, but ultimately they both stabilized the process and kept the melt pool area at its nominal value.

When the PW regime $(\delta=0.6)$ was adopted, the part was not sufficiently melted in the first layer before the control was applied, followed by a small overshoot of the melt pool area when the control tried to increase the duty cycle, before finally bringing and keeping the melt pool area on the steady-state value.

It was observed that the two schemes performed similarly, while the fixed reference point had a slightly faster response time compared to the control with adaptive reference point. Both strategies eventually stabilized the melting process. The control with fixed reference showed better results in terms of number of layers needed to bring the melt pool area to the reference value for the star geometry, indicating a more suitable condition for different geometries. Moreover, the simpler control scheme of the fixed reference point was found to be more appropriate to reduce the computational burden. With this conclusion, the layer-wise control strategy with fixed reference point was chosen to be implemented.

\section{VALIDATION OF THE CONTROL STRATEGY}

In order to validate the layer-wise control strategy, experiments were carried out on the 4-point star and the triangle shapes. Specimens without any control were also produced for comparison. Two replicates were produced for both geometries, each one consisting of 21 layers. The melt pool area was monitored and computed throughout each layer, and this information was used to correct the successive layers with the fixed reference point control strategy. The duty cycle employed in the first layer was equal to 1 for the entire layer. 3D melt pool maps were produced based on the experiment output. Focus variation microscopy was used on the produced specimens for a 3D reconstruction of the sample surfaces. Mean standard error of surface geometry was calculated from the reconstructions. The process parameters for the validation tests are reported in Table III. a)

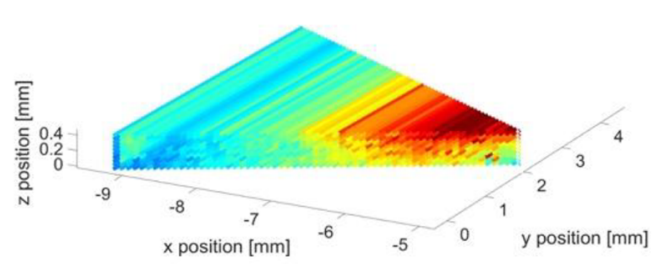

MPA $\left[\mathrm{mm}^{2}\right.$

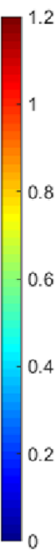

b)

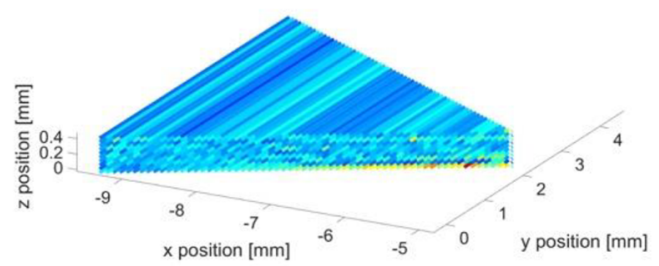

FIG. 10. Melt pool areas measured by the monitoring system during the production of the triangle-shaped specimens (a) without and (b) with the layer-wise control.

Figures 10 and 11 show the melt pool maps for triangle and star shapes without and with the layer-wise control. It can be seen that without the process control, the melt pool enlarges toward the pointy edges of the parts as the layer number

a)
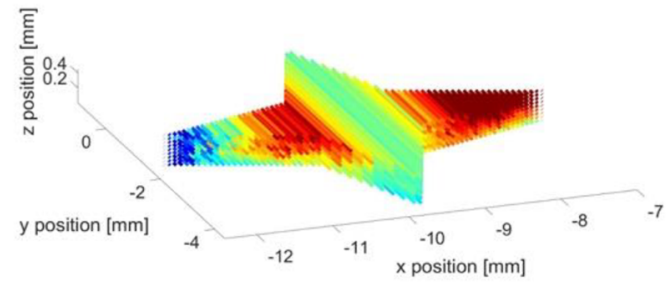

$\left.\mathrm{mm}^{2}\right]$

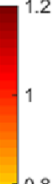

b)

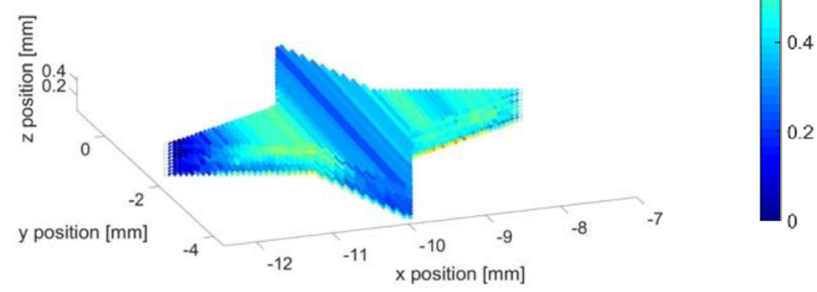

FIG. 11. Melt pool areas measured by the monitoring system during the production of the star-shaped specimens (a) without and (b) with the layer-wise control. 
a)

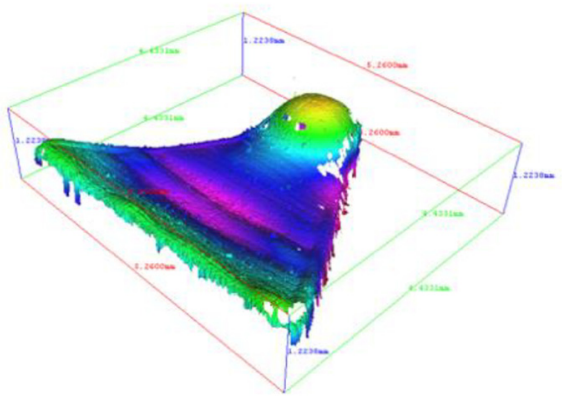

b)

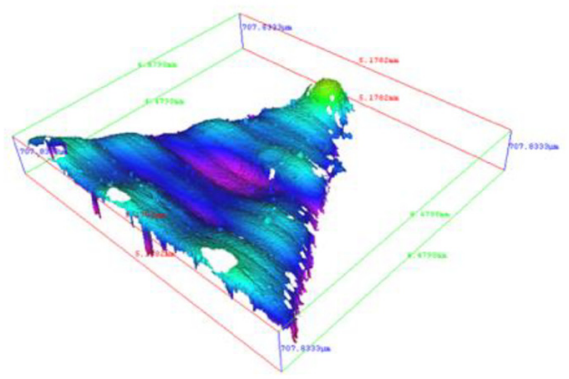

c)

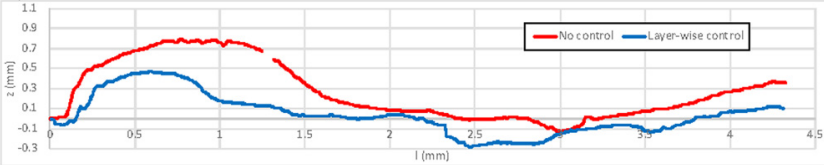

FIG. 12. 3D reconstructions of the produced triangle specimens (a) without $\left(\mathrm{MSE}=8.75 \mathrm{~mm}^{2}\right)$ and $(\mathrm{b})$ with the layer-wise control $\left(\mathrm{MSE}=1.54 \mathrm{~mm}^{2}\right)$. (c) Height map from perpendicular to the scan direction along the center.

progresses. The melt pool exceeds a value of $1 \mathrm{~mm}^{2}$ around the overheated regions corresponding to the last scanned vectors in both geometries.

Implementing the layer-wise control, it can be seen that a more homogeneous melt pool map can be obtained. It can be observed that the first layer of both the geometries show overheating regions. The use of the layer-wise control strategy successfully avoided the progression of the overheating regions along the successive layers. For both the geometries with the use of the layerwise control strategy, the melt pool could be maintained within the chosen MPA error margins.

Figures 12 and 13 report the 3D surface reconstructions of the produced specimens. It can be seen that for both of the geometries executed without the control scheme, severe swelling occurred around the edges with the smallest scan vectors. It can be observed that the highest swelling regions also correspond to the largest melt pool areas measured by the monitoring system.

The use of the layer-wise control appears to improve the swelling phenomenon significantly. In both geometries, the swelling around the pointy edges could be avoided. The $3 \mathrm{D}$ reconstructions showed that the mean square error could be lowered from 8.75 to
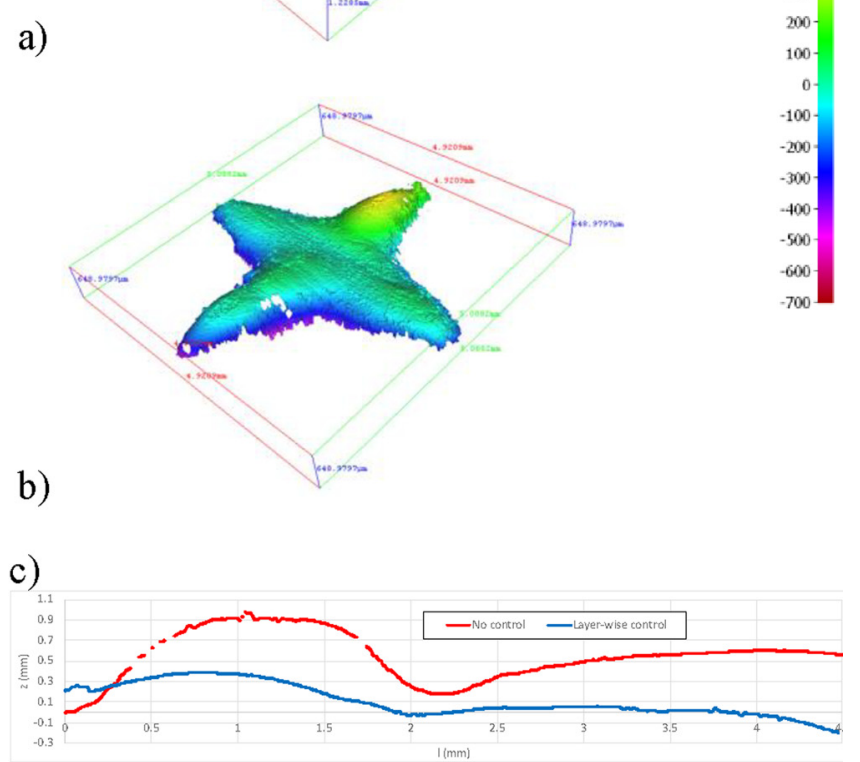

FIG. 13. 3D reconstructions of the produced star specimens (a) without $\left(M S E=6.42 \mathrm{~mm}^{2}\right)$ and $(b)$ with the layer-wise control $\left(M S E=1.19 \mathrm{~mm}^{2}\right)$. (c) Height map from perpendicular to the scan direction along the center.

$1.54 \mathrm{~mm}^{2}$ in the case of the triangle and from 6.42 to $1.19 \mathrm{~mm}^{2}$ for the star shape.

The results show the efficiency of the proposed method. However, several points remain unexplored. The use of a fixed scan direction remains an issue. The method requires further testing on different geometries with acute transition between the scan vector lengths. Moreover, the test geometries were characterized by a constant section along the build direction. The capacity of the strategy on geometries with variable sections should be further assessed. Finally, the response of the method when scanning overhang structures should be further studied.

\section{CONCLUSIONS}

The present work proposes a layer-wise control scheme for SLM to improve geometrical accuracy, in particular, by reducing hotspot formation and swelling phenomenon. The proposed method employs a coaxial melt-pool monitoring system that measures the melt pool area for each layer and corrects the energy 
density for the next layer by adjusting the duty cycle. The main outcomes of the work can be summarized as the following:

- A layer-wise control strategy can effectively be applied to control geometrical defects due to overheating.

- Heat accumulation is correlated to the scan vector length as the melt pool area enlarges around the thin sections.

- The duty cycle of the laser power can be effectively used to reduce the energy density without comprising the scan speed and hence productivity.

- The reaction time for settling to a stable melt pool area was found to be smaller via the use of a correction factor on the duty cycle of the previous layer $(\Delta \delta)$ rather than assigning a new duty cycle.

Future works will concentrate on the limitations regarding the use of the layer-wise control scheme. Particular attention will be paid on overheating causes which are not related to scan vector length, such as overhang regions. Evidently, the geometrical defects can be linked to metallurgical differences within the built part. The effect of the control strategies on metallurgical properties will also be assessed.

\section{ACKNOWLEDGMENTS}

The Italian Ministry of Education, University and Research is acknowledged for the support provided through the Project "Department of Excellence LIS4.0-Lightweight and Smart Structures for Industry 4.0."

\section{REFERENCES}

${ }^{1}$ M. Brandt, S. J. Sun, M. Leary, S. Feih, J. Elambasseril, and Q. C. Liu, "High-value SLM aerospace components: From design to manufacture," Adv. Mater. Res. 633, 135-147 (2013).

${ }^{2}$ M. K. Thompson, G. Moroni, T. Vaneker, G. Fadel, R. I. Campbell, I. Gibson, A. Bernard, J. Schulz, P. Graf, B. Ahuja, and F. Martina, "Design for additive manufacturing: Trends, opportunities, considerations, and constraints," CIRP Annals 65, 737-760 (2016).

${ }^{3} \mathrm{D}$. Wang et al., "Research on design optimization and manufacturing of coating pipes for automobile seal based on selective laser melting," J. Mater. Process. Technol. 273, 116227 (2019).

${ }^{4} \mathrm{M}$. Grasso and B. M. Colosimo, "Process defects and in situ monitoring methods in metal powder bed fusion: A review," Meas. Sci. Technol. 28, 044005 (2017).

${ }^{5}$ I. Baturynska, O. Semeniuta, and K. Martinsen, "Optimization of process parameters for powder bed fusion additive manufacturing by combination of machine learning and finite element method: A conceptual framework," Proc. CIRP 67, 227-232 (2018).

${ }^{6} \mathrm{~L}$. Scime and J. Beuth, "Using machine learning to identify in-situ melt pool signatures indicative of flaw formation in a laser powder bed fusion additive manufacturing process," Addit. Manuf. 25, 151-165 (2019).

${ }^{9}$ J. Fox, F. Lopez, B. Lane, H. Yeung, and S. Grantham, "On the requirements for model-based thermal control of melt pool geometry in laser powder bed fusion additive manufacturing," in Proceedings of the 2016 Material Science and
Technology Conference, MS\&T 2016, Salt Lake City, UT, 23-27 October 2016, pp. 133-140.

${ }^{7}$ L. Mazzoleni, A. G. Demir, L. Caprio, M. Pacher, and B. Previtali, "Real-time observation of melt pool in selective laser melting: Spatial, temporal, and wavelength resolution criteria," IEEE Trans. Instrum. Meas. 69, 1179-1190 (2020).

${ }^{8}$ M. Mani, S. Feng, B. Lane, A. Donmez, S. Moylan, and R. Fesperman, "Measurement science needs for real-time control of additive manufacturing powder-bed fusion processes," in Additive Manufacturing Handbook Product Development for the Defense Industry (CRC Press, Boca Raton, FL, 2017), pp. 629-676.

${ }^{10}$ J. P. Kruth, P. Mercelis, J. Van Vaerenbergh, and T. Craeghs, "Feedback control of selective laser melting," in Proceedings of the 3rd International Conference on Advanced Research in Virtual and Rapid Prototyping: Virtual and Rapid Manufacturing, Advanced Research Virtual and Rapid Prototyping, Leiria, Portugal, 17 September, 2007, pp. 521-527.

${ }^{11}$ T. Craeghs, F. Bechmann, S. Berumen, and J. P. Kruth, "Feedback control of layerwise laser melting using optical sensors," Phys. Proc. 5, 505-514 (2010).

${ }^{12}$ H. Yeung, B. M. Lane, M. A. Donmez, J. C. Fox, and J. Neira, "Implementation of advanced laser control strategies for powder bed fusion systems," Proc. Manuf. 26, 871-879 (2018).

${ }^{13}$ V. Renken, A. von Freyberg, K. Schünemann, F. Pastors, and A. Fischer, "In-process closed-loop control for stabilising the melt pool temperature in selective laser melting," Prog. Addit. Manuf. 4, 411-421 (2019).

${ }^{14}$ A. G. Demir, L. Mazzoleni, L. Caprio, M. Pacher, and B. Previtali, "Complementary use of pulsed and continuous wave emission modes to stabilize melt pool geometry in laser powder bed fusion," Opt. Laser Technol. 113, 15-26 (2019).

${ }^{15}$ T. Töppel, P. Schumann, M.-C. Ebert, T. Brokkes, K. Funke, M. Werner, F. Zeulner, and F. Bechmann, "3D analysis in laser beam melting based on realtime process monitoring," in Proceedings of the 2016 Material Science and Technology Conference, MS\&T 2016, Salt Lake City, UT, 23-27 October 2016, pp. 123-132.

${ }^{16}$ D. Alberts, D. Schwarze, and G. Witt, "High speed melt pool and laser power monitoring for selective laser melting," in 9th International Conference on Photonic Technologies LANE, Fürth, Germany, 19-22 September 2016.

${ }^{17}$ Renishaw, see https://www.renishaw.com/en/infiniam-spectral-42310 for "InfiniAM Spectral-Energy Input and Melt-Pool Emissions Monitoring for AM Systems" (accessed 12 June 2019).

${ }^{18}$ EOS, see https://www.eos.info/software/monitoring-software/ meltpool-monitoring for "EOSTATE MeltPool: Real-Time Process Monitoring for EOS M 290" (accessed 12 June 2019).

${ }^{19}$ M. Grasso, V. Laguzza, Q. Semeraro, and B. M. Colosimo, "In-process monitoring of selective laser melting: Spatial detection of defects via image data analysis," J. Manuf. Sci. Eng. 139, 051001 (2016).

${ }^{20}$ A. G. Demir, P. Colombo, and B. Previtali, "From pulsed to continuous wave emission in SLM with contemporary fiber laser sources: effect of temporal and spatial pulse overlap in part quality," Int. J. Adv. Manuf. Technol. 91, 2701-2714 (2017).

${ }^{21}$ M. Pacher, L. Mazzoleni, L. Caprio, A. G. Demir, and B. Previtali, "Estimation of melt pool size by complementary use of external illumination and process emission in coaxial monitoring of selective laser melting," J. Laser Appl. 31, 022305 (2019).

${ }^{22}$ L. Mazzoleni, L. Caprio, M. Pacher, and A. G. Demir, "External illumination strategies for melt pool geometry monitoring in SLM," JOM 71, 928-937 (2019). 